\title{
Energy Spectrum and Mass Composition of Ultra-High Energy Cosmic Rays Measured with the Telescope Array Fluorescence Detector Using a Monocular Analysis
}

\author{
Toshihiro Fujii ${ }^{*}, a, b$ for the Telescope Array Collaboration ${ }^{\dagger}$ \\ ${ }^{a}$ Institute for Cosmic Ray Research, University of Tokyo, Kashiwa, Chiba, 277-8583 JAPAN \\ ${ }^{b}$ Kavli Institute for Cosmological Physics, University of Chicago, Chicago, Ilinois, 60637 USA \\ E-mail: Eujiidkicp.uchicago.edu
}

\begin{abstract}
The Telescope Array (TA) experiment is the largest cosmic ray detector to observe ultra-high energy cosmic rays (UHECRs) in the northern hemisphere. We report on results of the energy spectrum of UHECRs covering a wide energy range, and the mass composition using the maximum shower depth, from analyzing data collected in monocular mode by the fluorescence detectors of TA during the first seven years.
\end{abstract}

The 34th International Cosmic Ray Conference,

30 July- 6 August, 2015

The Hague, The Netherlands

\footnotetext{
* Speaker.

${ }^{\dagger}$ Full author list: http://www.telescopearray.org/images/papers/ICRC2015-authorlist.pdf
} 


\section{Introduction}

In the research field of cosmic ray physics, one of the most intriguing topics is the origin of ultra-high energy cosmic rays (UHECRs) which are the most energetic particles in the universe. Because of an extremely low flux of UHECRs, a huge effective coverage is required to observe UHECRs. Measurements of the energy spectrum and the mass composition of UHECRs are essential for understanding origins and propagations of cosmic rays and for testing several theoretical models to produce UHECRs.

When UHECRs enter the Earth's atmosphere, a huge number of secondary particles are generated via hadronic and electromagnetic interactions with atmospheric nuclei, called an extensive air shower (EAS). The slant depth at which the longitudinal development of EAS reaches its maximum, $X_{\max }$, is an important parameter because it depends on the mass composition of UHECRs. A fluorescence detector (FD) observes atmospheric fluorescence photons emitted by molecules excited by an EAS, providing a determination of the primary energy and the longitudinal development of EAS including $X_{\max }$. This measurement has less dependence on simulations than other techniques such as the muon content of the EAS, because the production and energy-loss mechanisms of the EAS electromagnetic components (which make the dominant contribution to fluorescence photon emissions) are less dependent on hadronic interaction models.

The Telescope Array (TA) experiment is the largest cosmic ray detector in the northern hemisphere [四]. TA consists of 507 surface detectors (SDs) deployed on a square grid with $1.2 \mathrm{~km}$ spacing, covering an effective area of about $700 \mathrm{~km}^{2}$ [[]], overlooked by 38 fluorescence detectors at three locations [B]. One FD station located northwest of the SD array, consists each of 14 FDs previously used in the High Resolution Fly's Eye (HiRes) experiment [䧃]. Two other stations at the array's southeast and southwest consist of 12 newly designed and constructed FDs [B]], with with

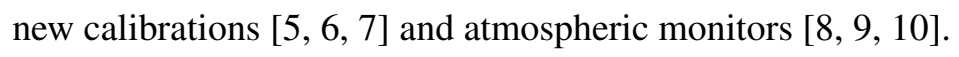

\section{Monocular Analysis and Monte Carlo Simulation}

In this paper, we report on an analysis of data collected at the newly constructed FD stations using a monocular analysis, which is an analysis mode to reconstruct an EAS to obtain properties of the primary particles using the measured shower image by one FD station. To reconstruct a geometry of an observed EAS, an arrival time $t_{i}$ of the signal in each photo-multiplier tube (PMT) $i$ is fitted by

$$
t_{i}=t_{\text {core }}+\frac{1}{c} \frac{\sin \Psi-\sin \alpha_{i}}{\sin \left(\Psi+\alpha_{i}\right)} r_{0}
$$

where $\alpha_{i}$ is an angle formed by the $i$-th PMT viewing direction and a direction vector from the FD station to the shower core (impact point of shower axis on the ground), $\Psi$ is the angle on the shower detector plane (SDP) formed by the shower axis and the direction to the shower core, $t_{\text {core }}$ is the time when the shower impacts the ground, and $r_{0}$ is the distance from the FD station to the shower core. When the EAS geometry has been determined, the longitudinal development of EAS is calculated by the inverse Monte Carlo method [U]]. This inverse Monte Carlo technique iteratively explores the longitudinal-development parameter space, searching for the optimum solution to reproduce the observed shower image. 

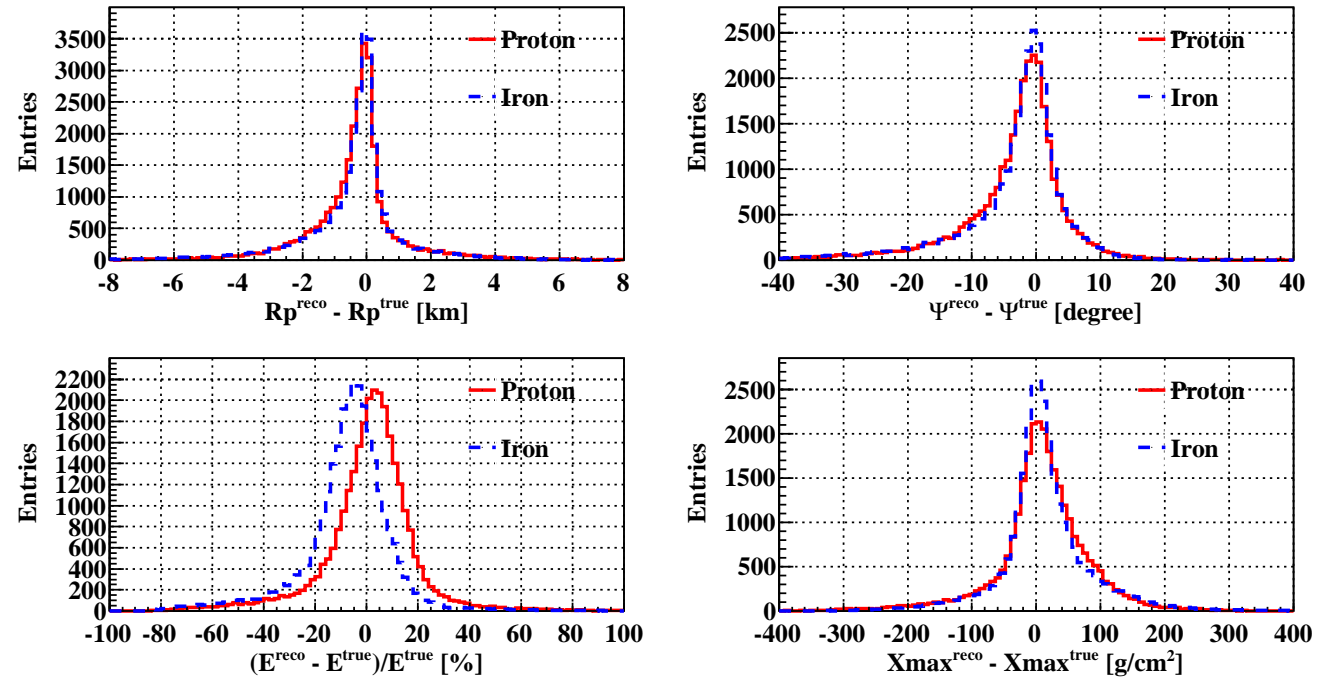

Figure 1: Resolutions of $R_{p}, P s i$, primary energy and $X_{\max }$ estimated by the reconstruction of artificial data using proton (red), and iron (blue dashed line) with the QGSJetII-03 interaction model in the monocular analysis.

The geometries of too faint or too short showers are difficult to reconstruct accurately. Thus, we apply quality cuts to select only well-reconstructed events in our analysis; the number of hit PMTs is larger than 10 , the track length is larger than $10^{\circ}$, the time extent is larger than $2 \mu \mathrm{s}$, and the depth of EAS maximum, $X_{\max }$, is within a field of view of FD, falling between the first and the last depths ( $X_{\text {start }}$ and $X_{\text {end }}$, respectively). To avoid the Cherenkov light contamination, we require the angle on the SDP is less than $120^{\circ}$ and the minimum viewing angle is greater than $20^{\circ}$.

The performance of the monocular analysis is estimated by the Monte Carlo (MC) detector simulation and reconstruction, comparing between the generated true parameters and reconstructed ones. Figure $\mathrm{W}$ shows the obtained resolution of the impact parameter $R_{p}$, angle on SDP $\Psi$, primary energy and $X_{\max }$ for proton and iron of the QGSJetII-03 interaction model in the CORSIKA software [[2]. As seen in Figure W, there is no large difference between proton and iron except for the reconstructed energy because the invisible energy for FD is corrected assuming a proton fraction reported by the HiRes/MIA experiment [[3] with the QGSJetII-03 model. The reconstructed energy of iron primary showers are underestimated about $6 \%$ because of the difference of the invisible energy. The resolutions of typical parameters in the monocular analysis are: $1.4 \mathrm{~km}$ on $R_{p}$, 7.7 degree on $\Psi$ angle, $17 \%$ on energy and $72 \mathrm{~g} / \mathrm{cm}^{2}$ on $X_{\max }$.

\section{Energy Spectrum Measurement}

To evaluate the energy spectrum of UHECRs, it is essential to calculate an aperture and an exposure of FD stations. The aperture cannot calculate a simple geometrical factor because it depends on not only the energies, but also the performance of FD, atmospheric models, PMT gains and primary shower species. Thus, we estimate the aperture of FD using MC simulations including these dependences. For the energy spectrum measurement, we use the QGSJetII-03 model to evaluate the aperture of the monocular analysis. The aperture of FD, $A \Omega$, is calculated 


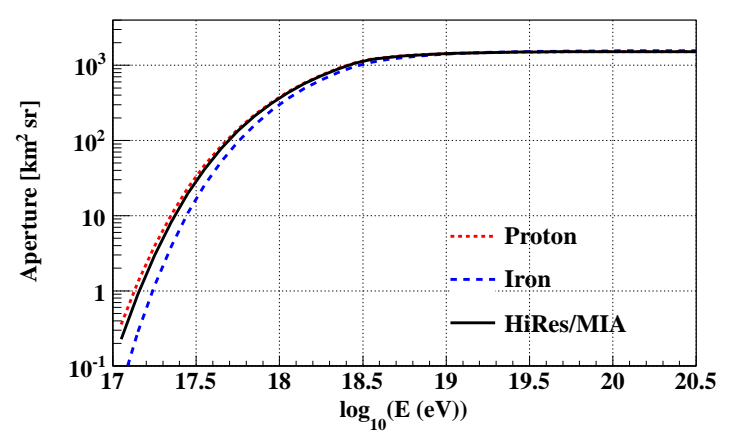

(a) Aperture of FD stations

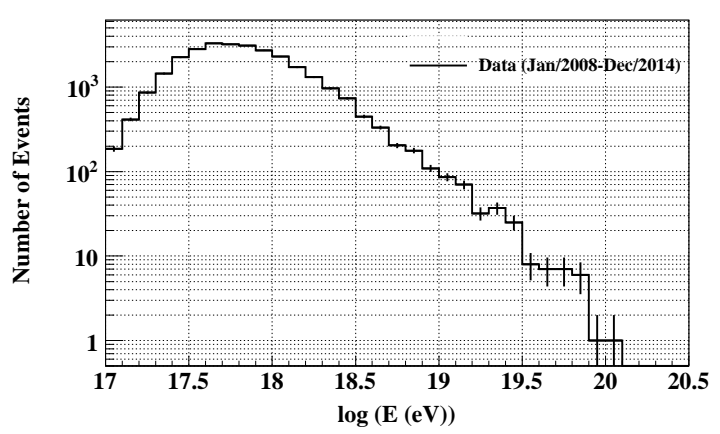

(b) Energy distribution of data

Figure 2: (a) The combined aperture of newly constructed FD stations evaluated by MC simulations for proton (red dotted line), iron (blue dashed line) and HiRes/MIA composition (black solid-line) in the monocular analysis. (b) Obtained energy distribution analyzing data observed by the newly constructed FD stations from January 2008 to December 2014.

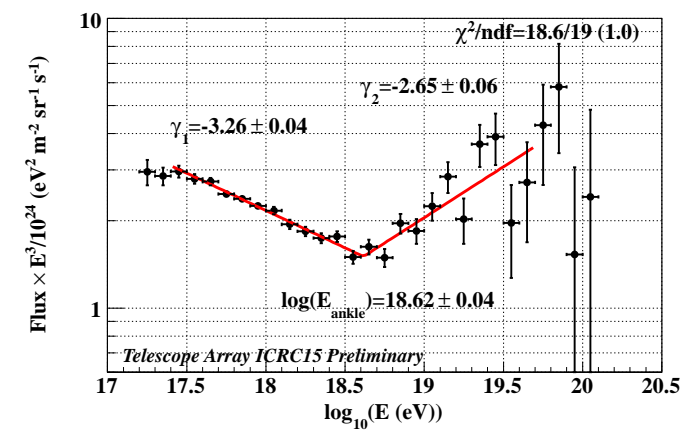

(a) Energy spectrum

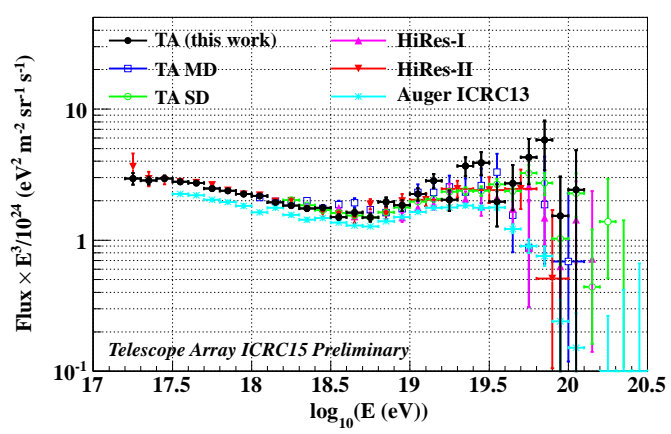

(b) Comparison with other results

Figure 3: (a) Energy spectrum observed by the newly constructed FD stations. (b) Energy spectrum compared with results reported by HiRes [四] and Auger [ए4] and other analyses of TA [ए5], ㅁ6].

from a ratio between the number of reconstructed events with the quality cuts and the number of thrown ones, $A \Omega(E)=A \Omega_{\text {gen }} \cdot N_{\text {reco }}(E) / N_{\text {thrown }}(E)$ where $E$ is the primary energy of cosmic ray, $A \Omega_{\mathrm{gen}}$ is the thrown aperture region of MC simulation, $N_{\text {reco }}$ is the number of reconstructed events and $N_{\text {thrown }}$ is the number of thrown events.

Since TA was designed for stereoscopic observations of air showers above $10^{19.0} \mathrm{eV}$, we define the combined aperture of the newly constructed FD stations in each monocular mode. When an energetic shower is reconstructed by the both stations, we select one result with a large number of photo-electrons to avoid the double counting of high energy showers. Using these reconstructed events by both stations, we estimate the combined aperture of newly constructed FD stations with proton, iron and HiRes/MIA reported composition [ए3] as shown in Fig. D(a). In the low energy region, the aperture is dependent on the primary species. In contrast, in the high energy region, it is independent of them.

Analyzing data collected from January 2008 to December 2014 using the monocular analy- 


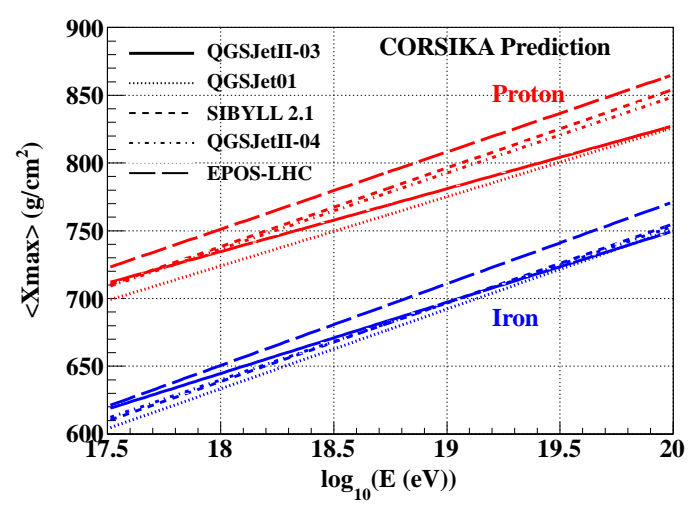

Figure 4: The average $X_{\max }$ of proton- and iron-induced (red and blue, respectively) showers generated by CORSIKA before detector simulation using QGSJet01 (dotted line), QGSJetII-03 (solid line), QGSJetII-04 (dash-dotted line), Sibyll 2.1 (short dashed line) and Epos-LHC (long dashed line).

sis, 28869 shower candidates above $10^{17} \mathrm{eV}$ are obtained as shown in Figure $\nabla(b)$. The energy spectrum is evaluated by the aperture assuming the HiRes/MIA composition, the number of events and the live time of FD. The obtained spectrum is shown with a single-broken power-law fitting in Figure B(a). There is an obvious broken energy at $\log \left(E_{\text {ankle }}\right)=18.62 \pm 0.04$ corresponding to the ankle structure. The total uncertainty on the energy scale is evaluated as $21 \%$ [ㅍ] ]. The obtained spectrum is compared with the other results reported by HiRes [田], Auger [U4] and other analyses of TA [15, [6] ]. The obtained spectrum is in good agreement with the HiRes-II spectrum in a broad energy region.

\section{Mass Composition Measurement}

For the mass composition measurement, we simulate shower developments of UHECRs using proton and iron, each according to five types of hadronic interaction models: QGSJet01, QGSJetII03, QGSJetII-04, Sibyll 2.1 and Epos-LHC in the CORSIKA software. Figure $\mathbb{t}$ shows the average values of the generated $X_{\max }$ as a function of the primary energy. As seen in Figure $\rightarrow$, the average $X_{\max }$ difference between proton and iron is $\sim 100 \mathrm{~g} / \mathrm{cm}^{2}$, with the choice of interaction model making at most difference of $\sim 25 \mathrm{~g} / \mathrm{cm}^{2}$ at $10^{17.5} \mathrm{eV}$ and $\sim 38 \mathrm{~g} / \mathrm{cm}^{2}$ at $10^{19.5} \mathrm{eV}$ for proton and $\sim 20 \mathrm{~g} / \mathrm{cm}^{2}$ for iron in the same energy range.

Since the FD has a limited field of view (FoV) and the $X_{\max }$ must be observed between $X_{\text {start }}$ and $X_{\text {end }}$, the observed $X_{\max }$ may be biased as a function of $X_{\text {start }}$ and $X_{\text {end }}$. In order to reduce the bias on $X_{\max }$, we define the biased $X_{\text {start }}$ and $X_{\text {end }}$ regions using the observed data and the MC simulations. We estimated that the fiducial FoV cuts on $X_{\text {start }}$ and $X_{\text {end }}$ are parameterized as $X_{\text {start }}(E) \leq 45.8 \times$ $\log (E)-215.8, X_{\text {end }}(E) \geq 20.8 \times \log (E)+501.4$ where $E(\mathrm{eV})$ is the reconstructed energy of UHECRs.

The fiducial FoV cuts and energy threshold are applied in the Monte Carlo detector simulation to evaluate expected average $X_{\max }$ and its distribution for proton or iron. Figure $\square$ shows the distribution of observed $X_{\max }$ and expected ones in several energy bins. The expected distributions for three different mass compositions are shown for comparison: pure proton, pure iron, or an equal 

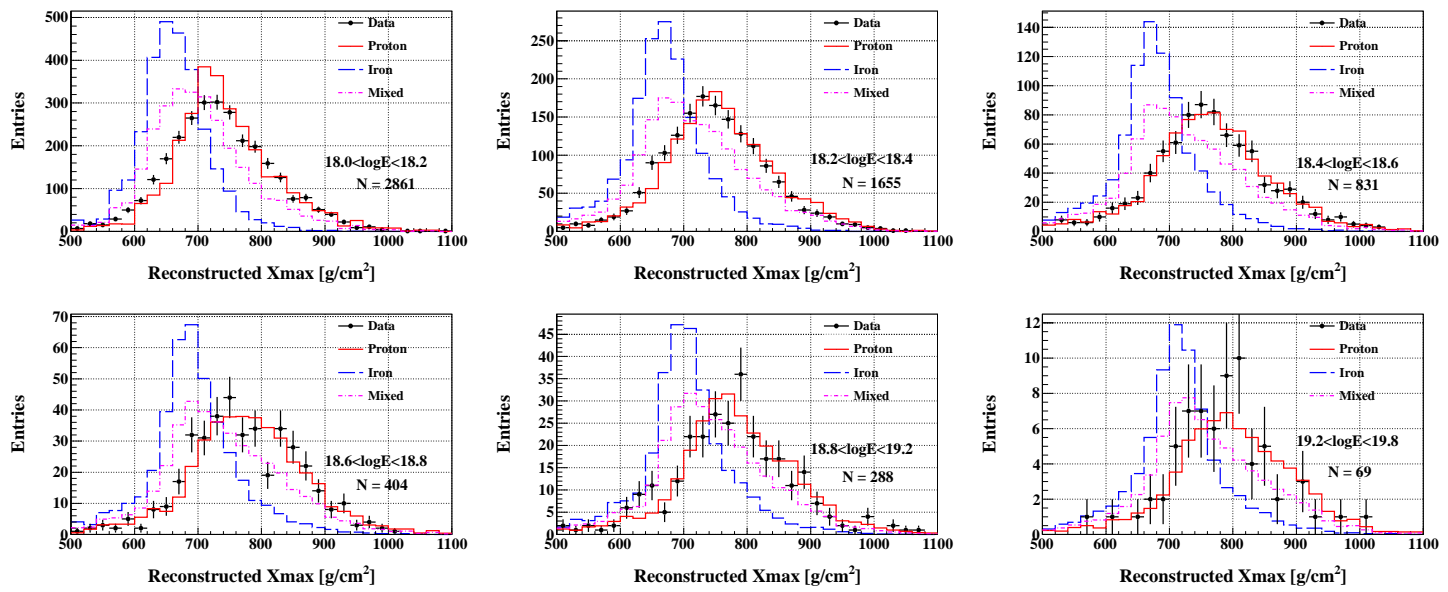

Figure 5: $X_{\max }$ distributions in each energy range using the fiducial FoV cuts, compared with the expected distributions estimated from MC simulations using QGSJetII-03 with three different compositions: pure proton (red solid line), pure iron (blue dashed line), and a equal mixture of both (pink dash-dotted line).

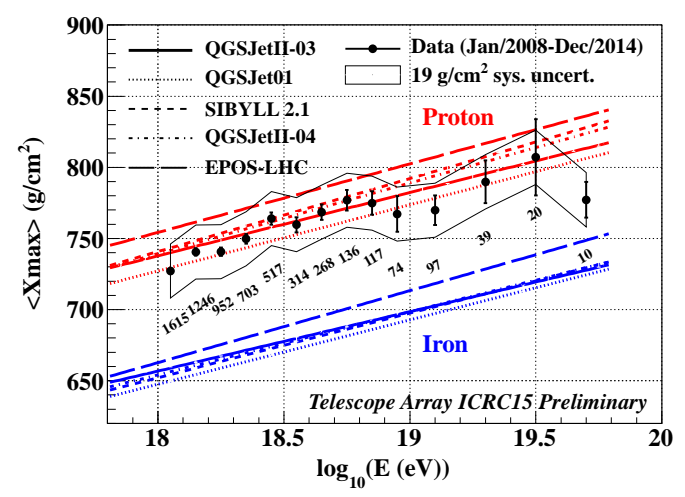

(a) Obtained $<X_{\max }>$ compared with MC predictions

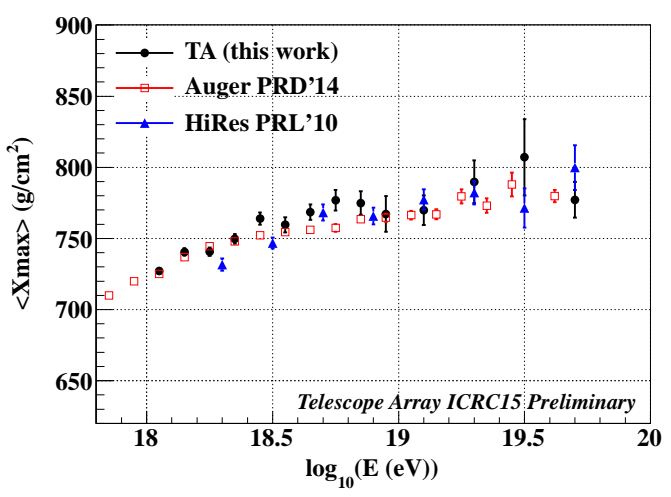

(b) $<X_{\max }>$ compared with other results

Figure 6: (a) Evolution of the average $X_{\max }$ with energy from data (black points), plotted with the MC simulation results (lines) for two particle species (proton, iron) and five hadronic interaction models. The box region shows the systematic uncertainty on $X_{\max }, 19 \mathrm{~g} / \mathrm{cm}^{2}$, for the monocular analysis. (b) Average

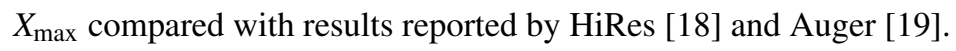

mixture of both using the best matched model of QGSJetII-03 model. Figure G(a) shows the comparison between observed $X_{\max }$ and expected $X_{\max }$ estimated by the Monte Carlo simulations. The systematic uncertainty on $<X_{\max }>$ is $19 \mathrm{~g} / \mathrm{cm}^{2}$ for the monocular analysis indicating the box region in this figure. The uncertainty includes the fluorescence yield $\left(5 \mathrm{~g} / \mathrm{cm}^{2}\right)$, the atmospheric condition $\left(12 \mathrm{~g} / \mathrm{cm}^{2}\right)$, the FD calibration $\left(5 \mathrm{~g} / \mathrm{cm}^{2}\right)$, the FD geometry $\left(9 \mathrm{~g} / \mathrm{cm}^{2}\right)$ and the shower reconstruction $\left(10 \mathrm{~g} / \mathrm{cm}^{2}\right)$. The obtained average $X_{\max }$ and its distributions indicate proton-dominated composition at this energy range which is consistent with results already reported by TA stereo or hybrid analyses [20, [2]]. The obtained $X_{\max }$ is compared with results reported by HiRes [[1]] and Auger [प्व]] as shown in Figure $\mathbf{G}(\mathrm{b})$. Those results show in good agreement within the systematic uncertainty. 


\section{Conclusions}

We report on a measurement of the cosmic ray spectrum covering a broad range of energies above $10^{17.2} \mathrm{eV}$ analyzed by the newly constructed fluorescence detectors of the Telescope Array experiment using the monocular analysis during the first seven years. The obtained spectrum has an obvious broken structure at energy of $\log \left(E_{\text {ankle }}\right)=18.62 \pm 0.04$. The structure is in good agreement with the spectra reported using the TA surface detector, and by the HiRes-II. We report the mass composition using the fiducial FoV cuts to reduce observation bias on $X_{\max }$. The obtained average $X_{\max }$ and its distribution shows proton-dominated composition at this energy range which is consistent with already reported results within the systematic uncertainty.

\section{Acknowledgements}

The Telescope Array experiment is supported by the Japan Society for the Promotion of Science through Grants-in-Aid for Scientific Research on Specially Promoted Research (21000002) "Extreme Phenomena in the Universe Explored by Highest Energy Cosmic Rays" and for Scientific Research (19104006), and the Inter-University Research Program of the Institute for Cosmic Ray Research; by the U.S. National Science Foundation awards PHY-0307098, PHY-0601915, PHY-0649681, PHY-0703893, PHY-0758342, PHY-0848320, PHY-1069280, PHY-1069286, PHY1404495 and PHY-1404502; by the National Research Foundation of Korea (2007-0093860, R3210130, 2012R1A1A2008381, 2013004883); by the Russian Academy of Sciences, RFBR grants 11-02-01528a and 13-02-01311a (INR), IISN project No. 4.4502.13, and Belgian Science Policy under IUAP VII/37 (ULB). The foundations of Dr. Ezekiel R. and Edna Wattis Dumke, Willard L. Eccles, and George S. and Dolores Doré Eccles all helped with generous donations. The State of Utah supported the project through its Economic Development Board, and the University of Utah through the Office of the Vice President for Research. The experimental site became available through the cooperation of the Utah School and Institutional Trust Lands Administration (SITLA), U.S. Bureau of Land Management, and the U.S. Air Force. We also wish to thank the people and the officials of Millard County, Utah for their steadfast and warm support. We gratefully acknowledge the contributions from the technical staffs of our home institutions. An allocation of computer time from the Center for High Performance Computing at the University of Utah is gratefully acknowledged.

\section{References}

[1] Telescope Array Collaboration, M. Fukushima et al., Telescope array project for extremely high energy cosmic rays, Prog.Theor.Phys.Suppl. 151 (2003) 206-210.

[2] Telescope Array Collaboration, T. Abu-Zayyad et al., The surface detector array of the Telescope Array experiment, Nucl.Instrum.Meth. A689 (2012) 87-97, [arXiv: 1201.4964].

[3] H. Tokuno, Y. Tameda, M. Takeda, K. Kadota, D. Ikeda, et al., New air fluorescence detectors employed in the Telescope Array experiment, Nucl.Instrum.Meth. A676 (2012) 54-65, [arXiv: 1201.0002].

[4] HiRes Collaboration, R. Abbasi et al., First observation of the Greisen-Zatsepin-Kuzmin suppression, Phys.Rev.Lett. 100 (2008) 101101, [astro-ph/0703099]. 
[5] S. Kawana, N. Sakurai, T. Fujii, M. Fukushima, N. Inoue, et al., Calibration of Photomultiplier Tubes for the Fluorescence Detector of Telescope Array Experiment using a Rayleigh Scattered Laser Beam, Nucl.Instrum.Meth. A681 (2012) 68-77, [arXiv:1202.1934]].

[6] H. Tokuno, Y. Murano, S. Kawana, Y. Tameda, A. Taketa, et al., On site calibration for new fluorescence detectors of the telescope array experiment, Nucl.Instrum.Meth. A601 (2009) 364-371.

[7] B. K. Shin, H. Tokuno, Y. Tsunesada, T. Abu-Zayyad, R. Aida, et al., Gain monitoring of telescope array photomultiplier cameras for the first 4 years of operation, Nucl.Instrum.Meth. A768 (2014) 96-103.

[8] S. Udo, R. Cady, M. Fukushima, J. Matthews, T. Jason, et al., The Central Laser Facility at the Telescope Array, Proc. of the 30th International Cosmic Ray Conference, Merida, Mexico 5 (2007) 1021-1024.

[9] T. Tomida, Y. Tsuyuguchi, T. Arai, T. Benno, M. Chikawa, et al., The atmospheric transparency measured with a LIDAR system at the Telescope Array experiment, Nucl.Instrum.Meth. $\mathbf{A 6 5 4}$ (2011) 653-660,

[10] Telescope Array Collaboration, T. Tomida et al., Atmospheric monitor for Telescope Array experiment, EPJ Web Conf. 53 (2013) 10003.

[11] Telescope Array Collaboration, T. Fujii et al., An event reconstruction method for the Telescope Array Fluorescence Detectors, AIP Conf.Proc. 1367 (2011) 149-152.

[12] D. Heck, G. Schatz, T. Thouw, J. Knapp, and J. Capdevielle, CORSIKA: A Monte Carlo code to simulate extensive air showers, Forschungszentrum Karlsruhe Report FZKA (1998) 6019.

[13] HiRes-MIA Collaboration, T. Abu-Zayyad et al., Measurement of the cosmic ray energy spectrum and composition from $10^{17} \mathrm{eV}$ to $10^{18.3} \mathrm{eV}$ using a hybrid fluorescence technique, Astrophys.J. 557 (2001) 686-699, [astro-ph/0010652].

[14] Pierre Auger Collaboration, A. Schulz, The measurement of the energy spectrum of cosmic rays above $3 \times 10^{17} \mathrm{eV}$ with the Pierre Auger Observatory, Proc. of the 33rd International Cosmic Ray Conference, Rio de Janeiro, Brazil (2013) [arXiv: 1307.5059].

[15] Telescope Array Collaboration, D. Ivanov, TA Spectrum Summary, Proc. of the 34th International Cosmic Ray Conference, Hague, Netherlands (2015) ID349.

[16] Telescope Array Collaboration, T. Abu-Zayyad et al., The Energy Spectrum of Telescope Array's Middle Drum Detector and the Direct Comparison to the High Resolution Fly's Eye Experiment, Astropart.Phys. 39-40 (2012) 109-119, [arXiv:1202.5141].

[17] Telescope Array Collaboration, Y. Tsunesada et al., TA Energy Scale: Methods and Photometry, Proc. of the 32th International Cosmic Ray Conference, Beijing, China (2011) ID1270.

[18] HiRes Collaboration, R. Abbasi et al., Indications of Proton-Dominated Cosmic Ray Composition above 1.6 EeV, Phys.Rev.Lett. 104 (2010) 161101, [arxiv:0910.4184]].

[19] Pierre Auger Collaboration, A. Aab et al., Depth of maximum of air-shower profiles at the Pierre Auger Observatory. I. Measurements at energies above $10^{17.8} \mathrm{eV}$, Phys.Rev. D90 (2014) 122005, [arxiv:]409.4809].

[20] Telescope Array Collaboration, Y. Tameda, Measurement of UHECR composition by TA, AIP Conf.Proc. 1367 (2011) 110-113.

[21] Telescope Array Collaboration, R. Abbasi, M. Abe, T. Abu-Zayyad, M. Allen, R. Anderson, et al., Study of Ultra-High Energy Cosmic Ray composition using Telescope Array' s Middle Drum detector and surface array in hybrid mode, Astropart.Phys. 64 (2014) 49-62, 